\title{
Psychiatrists' self-stigma, the DGPPN guideline for psychosocial interventions, and contemporary treatment strategies
}

\author{
Daniela Reich-Erkelenz • Andrea Schmitt • Peter Falkai
}

Published online: 24 February 2015

(C) Springer-Verlag Berlin Heidelberg 2015

Gaebel et al. [1] summarize the results of a survey conducted by the WPA task force on destigmatization, which assessed the extent to which the stigma of mental illness affects psychiatrists compared to general practitioners in 12 countries. The questionnaire presented to 1893 psychiatrists and 1238 general practitioners comprised perceived stigma, self-stigma (stereotype agreement), attitudes toward the other profession and experienced discrimination. Compared to general practitioners, psychiatrists reported significantly higher stigma and discrimination experiences. Additionally, perceived stigma in psychiatrists correlated best with discrimination experiences and self-stigma, whereas in general practitioners with self-stigma alone. Moreover, about $17 \%$ of, by the majority, younger psychiatrists regarded stigma as a serious problem of their profession. Thus, existing antistigma interventions for mental health professionals and students need to be enhanced to raise awareness of own stigmatizing attitudes and to improve professional-related self-assertiveness.

Summarizing the recently published DGPPN guideline on psychosocial therapies, Gühne et al. [2] present new findings based on systematic literature searches about best treatment options for people with severe mental illness. This guideline distinguishes between system-level and single psychosocial interventions and stresses the evidence of the efficacy of multidisciplinary team-based psychiatric

D. Reich-Erkelenz

Institute of Psychiatric Phenomics and Genomics,

Ludwig-Maximilians-University Munich, Munich, Germany

A. Schmitt $(\bowtie) \cdot$ P. Falkai

Department of Psychiatry and Psychotherapy,

Ludwig-Maximilians-University Munich, Nußbaumstr. 7,

80336 Munich, Germany

e-mail: Andrea.Schmitt@med.uni-muenchen.de community care, family psychoeducation, social skills training, and supported employment. Anyhow, there still is a need for further context-specific research in severe mental illness. Holl et al. [3], again, focus on the abidance of recommendations on pharmacological treatment of internationally published treatment guidelines of anxiety disorders. Screening data of 2573 psychiatric inpatients with the respective diagnosis (phobia, panic disorder, generalized anxiety disorder, other diagnosis of anxiety) filed in the international drug safety program in psychiatry (AMSP), the authors found that all diagnostic groups were only partially treated in accordance with international treatment guidelines, for which reason they see a strong need for a better implementation of recommendations in primary and secondary care. Aiming on reducing compulsory psychiatric admissions, Lay et al. [4] conducted a study in 238 patients with severe mental illness who had at least one compulsory admission within the past 24 months. For the duration of 12 months, participants were randomly assigned to individualized psychoeducation or merely treatment as usual. At the end of study, $35.4 \%$ of patients with treatment as usual, but only $22.5 \%$ patients of the intervention group had been compulsorily readmitted to psychiatry bespeaking a significant effectiveness of psychoeducation as add-on treatment in preventing compulsory readmissions.

In their ${ }^{1} \mathrm{H}$-magentic resonance spectroscopy study, Tükel et al. [5] investigated the effects of SSRI treatment on the neurochemical levels in patients with obsessive-compulsive disorder (OCD). The authors specifically measured $N$-acetylaspartate (NAA), choline, and myo-inositol levels in terms of their ratios with creatine in both 19 patients with 12 weeks of sertraline treatment and healthy controls. At baseline, OCD patients showed significantly lower NAA/Cr levels in the anterior cingulate and in the 
caudate compared to controls, but no such differences after 12 weeks of sertraline treatment, which raised NAA/Cr levels significantly. Thus, NAA reductions seem to be reversible via SSRI treatment, which possibly improves neuronal integrity. Since a potential relation between peripheral biological markers and the seizure quality of ECT as surrogate for treatment efficacy has yet not been investigated, Bumb et al. [6] in their study prospectively examined BDNF levels in 20 patients with major depression before and after ECT. The authors found not only BDNF levels to be significantly higher after ECT, but also a significant correlation of serum BDNF levels and time between the last ECT and blood withdrawal. These findings reinforce the neurotrophin hypothesis, namely that ECT-induced central BDNF rise leads to a delayed and increased equilibrium of the peripheral BDNF, which underlines the importance of monitoring seizure quality markers in daily practice. After the recent approval of methylphenidate (MPH), Antel et al. [7] expect an enormous increase in the number of ADHD patients treated with this drug in the foreseeable future. Although two large retrospective cohort studies on serious cardiovascular events in children and adolescents came to the point that MPH was safe in the short- and medium-term use, the authors refer to the inherent limitations of retrospective cohort studies, especially considering the lately discovered risks of the similarly acting sibutramine with its comparable side effects on heart rate and blood pressure. In the interest of an optimized risk assessment, they plead for conducting a prospective randomized, double-blind, placebo-controlled, multicenter trial like SCOUT in persons stratified for cardiovascular risk factors.

In their meta-analysis of six RCTs with only schizophrenic $(N=352)$ and one with also bipolar disorder patients $(N=77)$, Kishi and Iwata [8] investigated the effects of varenicline adjuvant therapy for smoking cessation in schizophrenia patients. According to their analysis, varenicline was superior to placebo neither in smoking cessation nor for overall, positive, negative, and depressive symptoms. They furthermore found no significant differences in the discontinuation rate due to any causes, clinical deterioration, side effects, suicidal ideation, and depression between varenicline of placebo. The only slightly visible effect of varenicline compared to placebo consisted in a lower rate of abnormal dreams/nightmares. Thus, varenicline adjuvant therapy seems not to be superior to placebo, which should be further investigated in future studies with larger samples. Smoking reduction or cessation was also subject of the study by Chou et al. [9], since readiness to quit as predictor for smoking cessation has numerously been studied in the general population, but not so far in schizophrenia patients. The authors included 308 schizophrenic inpatients using nicotine replacement therapy or bupropion and categorized them into stages of change (no immediate intention to stop, intention to quit in the next 6 months, intention to quit in the next month). They found a significant difference in change in number of cigarettes (NOC) and Fagerstrom test for nicotine dependence (FTND) score plus a trend of increasing reduction in NOC and FTND scores in different stages of change, but no significant association between stage of change and smoking cessation, which the authors ascribe to the very small sample of successful quitters. In summary, stage of change could predict smoking reduction and thus could be a useful indicator for patients' preparedness before trying to reduce NOC.

\section{References}

1. Gaebel W, Zäske H, Zielasek J, Cleveland H-R, Samjeske K, Stuart H, Arboleda-Florez J, Akiyama T, Baumann AE, Gureje O, Jorge M, Kastrup MR, Suzuki Y, Tasman A, Fidalgo TM, Jarema M, Johnson SB, Kola L, Krupchanka D, Larach V, Matthews L, Mellsop G, Ndetei DM, Okasha TA, Padalko E, Spurgeoun JA, Tyszkowska M, Sartorius N (2014) Stigmatization of psychiatrists and general practitioners: results of an international survey. Eur Arch Psychiatry Clin Neurosci. doi:10.1007/ s00406-014-0530-8

2. Gühne U, Weinmann S, Arnold K, Becker T, Riedel-Heller SG (2014) S3 guideline on psychosocial therapies in severe mental illness: evidence and recommendations. Eur Arch Psychiatry Clin Neurosci. doi:10.1007/s00406-014-0558-9

3. Holl AK, Grohmann R, Letmaier M, Painold A, Mörkl S, Toto S, Kasper S (2014) Pharmacotherapy of anxiety disorders in German-speaking countries: current status and changes between 1994 and 2011. Eur Arch Psychiatry Clin Neurosci. doi:10.1007/ s00406-014-0523-7

4. Lay B, Blank C, Lengler S, Drack T, Bleiker M, Rössler W (2014) Preventing compulsory admission to psychiatric inpatient care using psycho-education and monitoring: feasibility and outcomes after 12 months. Eur Arch Psychiatry Clin Neurosci. doi:10.1007/s00406-014-0553-1

5. Tükel R, Aydın K, Ertekin E, Özyıldırım S, Barburoğlu M (2014) ${ }^{1} \mathrm{H}$-magnetic resonance spectroscopy in obsessive-compulsive disorder: effects of 12 weeks of sertraline treatment on brain metabolites. Eur Arch Psychiatry Clin Neurosci. doi:10.1007/ s00406-014-0545-1

6. Bumb J, Suna S, Aksay C, Kranaster L, Geisel O, Gass P, Hellweg R, Sartorius A (2014) Focus on ECT seizure quality: serum BDNF as a peripheral biomarker in depressed patients. Eur Arch Psychiatry Clin Neurosci. doi:10.1007/s00406-014-0543-3

7. Antel J, Albayrak Ö, Heusch G, Banaschewski T, Hebebrand J (2014) Assessment of potential cardiovascular risks of methylphenidate in comparison with sibutramine: do we need a SCOUT (trial)? Eur Arch Psychiatry Clin Neurosci. doi:10.1007/ s00406-014-0522-8

8. Kishi T, Iwata N (2014) Varenicline for smoking cessation in people with schizophrenia: systematic review and meta-analysis. Eur Arch Psychiatry Clin Neurosci. doi:10.1007/s00406-014-0551-3

9. Chou K-J, Chen H-K, Hung C-H, Chen T-T, Chen C-M, BoJian W (2014) Readiness to quit as a predictor for outcomes of smoking-reduction programme with transdermal nicotine patch or bupropion in a sample of 308 patients with schizophrenia. Eur Arch Psychiatry Clin Neurosci. doi:10.1007/s00406-014-0515-7 